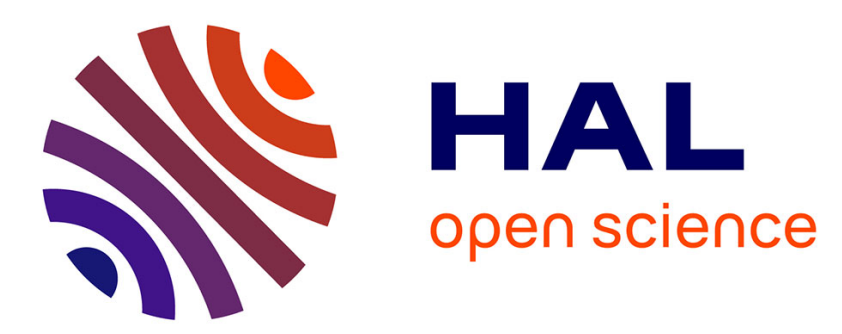

\title{
An Interpolated Model Recovery Anti-Windup for a Canard-Guided Projectile Subject to Uncertainties
}

Sovanna Thai, Clément Roos, Jean-Marc Biannic, Spilios Theodoulis

\section{To cite this version:}

Sovanna Thai, Clément Roos, Jean-Marc Biannic, Spilios Theodoulis. An Interpolated Model Recovery Anti-Windup for a Canard-Guided Projectile Subject to Uncertainties. IEEE ECC 2021, Jun 2021, VIRTUEL, Netherlands. hal-03523362

\section{HAL Id: hal-03523362 https://hal.science/hal-03523362}

Submitted on 12 Jan 2022

HAL is a multi-disciplinary open access archive for the deposit and dissemination of scientific research documents, whether they are published or not. The documents may come from teaching and research institutions in France or abroad, or from public or private research centers.
L'archive ouverte pluridisciplinaire HAL, est destinée au dépôt et à la diffusion de documents scientifiques de niveau recherche, publiés ou non, émanant des établissements d'enseignement et de recherche français ou étrangers, des laboratoires publics ou privés. 


\title{
An Interpolated Model Recovery Anti-Windup for a Canard-Guided Projectile Subject to Uncertainties
}

\author{
Sovanna Thai ${ }^{1}$, Clément Roos $^{2}$, Jean-Marc Biannic ${ }^{2}$, and Spilios Theodoulis ${ }^{1}$
}

\begin{abstract}
This paper presents an autopilot design for a dualspin guided projectile subject to aerodynamic uncertainties and actuator saturations. The proposed design consists of a gain-scheduled baseline controller together with an interpolated dynamic anti-windup compensator based on model recovery. Evaluation of the closed-loop is done through IQC-based analysis at an operating point, and through Monte Carlo simulations. Both assessments show that the addition of an antiwindup compensator can drastically improve the behaviour of the system in degraded flight conditions.
\end{abstract}

Index Terms-guided projectiles, autopilot design, gain scheduling, anti-windup, robustness analysis

\section{INTRODUCTION}

The unguided nature of ballistic projectiles makes them incompatible with demanding operational requirements. Indeed, deviations from the nominal trajectory can easily occur due to disturbances or uncertainties, ultimately leading to significant miss distances. Guided projectile concepts seek to overcome these shortcomings by adding steering mechanisms.

The concept studied in this paper is a dual-spin projectile featuring a roll-decoupled nose equipped with four canards (see Fig.1). These canards are independently actuated, and their deflection allows to alter the trajectory in flight. A standard flight scenario of such a projectile is depicted in Fig.2. During the ballistic phase, the on-board electronics activate, and a roll autopilot ensures control of the nose position. Afterwards, the guided phase starts, during which additional efforts on the pitch and yaw channels are generated by the canards. Although attractive for its apparent simplicity, this solution leads to a challenging control problem. Previous work on this concept include [1], which tackled system modelling as well as controller synthesis. More recently in [2], saturations were taken into account and a gain-scheduled static anti-windup compensator was proposed. Building up on these previous studies, this paper revisits the anti-windup design and proposes a dynamic compensator with a suitable interpolation strategy, and sets up the integral quadratic constraints (IQC) framework for robustness analysis of the closed-loop against both aerodynamic uncertainties and saturations.

\footnotetext{
${ }^{1}$ Sovanna Thai and Spilios Theodoulis are with the Guidance, Navigation \& Control Department of the French-German Research Institute of Saint-Louis (ISL), Saint-Louis,France <sovanna.thai, spilios.theodoulis>isl.eu

${ }^{2}$ Clément Roos and Jean-Marc Biannic are with the Information Processing and Systems Department of ONERA, The French Aerospace Lab, Toulouse, France <clement.roos, jean-marc.biannic>@onera.fr
}

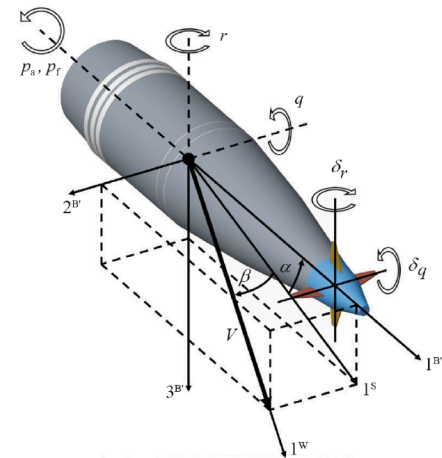

Fig. 1. Dual-spin projectile concept

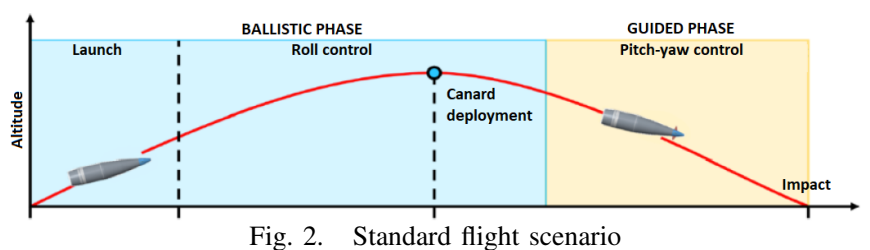

Section II reviews the open-loop components and model. Section III deals with autopilot design, with a brief description of the baseline autopilot from [2], and the computation of a dynamic anti-windup compensator. Section IV presents the robustness analysis framework and early analysis results, and Section V details Monte Carlo simulations.

\section{OPEN-LOOP MOdEL}

The 7 degree-of-freedom airframe model is described by its translational and attitude dynamic equations. They are expressed in a non-rolling reference frame $B^{\prime}$ shown in Fig. 1 as:

$$
\begin{aligned}
& {\left[\begin{array}{c}
\dot{u} \\
\dot{v} \\
\dot{w}
\end{array}\right] }=\frac{1}{m}\left[\begin{array}{c}
X \\
Y \\
Z
\end{array}\right]-\left[\begin{array}{ccc}
0 & -r & q \\
r & 0 & r \tan \theta \\
-q & -r \tan \theta & 0
\end{array}\right]\left[\begin{array}{c}
u \\
v \\
w
\end{array}\right] \\
& {\left[\begin{array}{c}
\dot{p}_{f} \\
\dot{p}_{a} \\
\dot{q} \\
\dot{r}
\end{array}\right]=\left[\begin{array}{c}
I_{x f}{ }^{-1} L_{f} \\
I_{x a}{ }^{-1} L_{a} \\
I_{t}{ }^{-1} M \\
I_{t}{ }^{-1} N
\end{array}\right]+\left[\begin{array}{c}
0 \\
-\left(I_{x a} I_{t}^{-1} p_{a}+r \tan \theta\right) r \\
\left(I_{x a} I_{t}{ }^{-1} p_{a}+r \tan \theta\right) q
\end{array}\right] }
\end{aligned}
$$

where $[u, v, w]^{T}$ are the linear velocities, and $\left[p_{f}, p_{a}, q, r\right]^{T}$ the angular rates of the projectile (the subscripts $f$ and $a$ denote the forward and aft parts respectively). The constants appearing in these equations are the mass $m$ of the projectile and its moments of inertia $I_{x f}, I_{x a}$, and $I_{t}$. Complementing these equations are the translational and attitude kinematic equations which describe the linear and angular positions, $\left[x_{e}, y_{e}, z_{e}\right]^{T}$ and $\left[\phi_{f}, \phi_{a}, \theta, \psi\right]^{T}$, with respect to the inertial 
reference frame. These are omitted for brevity but can be found e.g. in [1].

Assuming no wind, Eq. (1a) can be rewritten in terms of the airframe velocity $V$, angle of attack $\alpha$, and angle of sideslip $\beta$, in a form that is more useful for control design purposes:

$$
\begin{aligned}
{\left[\begin{array}{c}
\dot{V} \\
\dot{\alpha} \\
\dot{\beta}
\end{array}\right] } & =\left[\begin{array}{c}
0 \\
q+r(\cos \alpha \tan \theta-\sin \alpha) \tan \beta \\
-r(\cos \alpha+\sin \alpha \tan \theta)
\end{array}\right] \\
& +\frac{1}{m V}\left[\begin{array}{ccc}
V \cos \alpha \cos \beta & V \sin \beta & V \sin \alpha \cos \beta \\
-\sin \alpha / \cos \beta & 0 & \cos \alpha / \cos \beta \\
-\cos \alpha \sin \beta & \cos \beta & -\sin \alpha \sin \beta
\end{array}\right]\left[\begin{array}{c}
X \\
Y \\
Z
\end{array}\right]
\end{aligned}
$$

The modelling of the forces and moments is based on ballistics theory as presented in [3]. For brevity, we focus on the pitch/yaw channels, as the roll channel is not as relevant for autopilot design. The external forces $Y, Z$ include lift, Magnus (originating from the spinning motion), control (generated by the canards), and gravitational terms:

$$
\begin{aligned}
{\left[\begin{array}{l}
Y \\
Z
\end{array}\right] } & =\bar{q} S\left(\left[\begin{array}{l}
-C_{N \alpha}(\mathcal{M}, \alpha, \beta) \beta \\
-C_{N \alpha}(\mathcal{M}, \alpha, \beta) \alpha
\end{array}\right]+\frac{p_{a} d}{2 V}\left[\begin{array}{l}
+C_{y p \alpha}(\mathcal{M}) \alpha \\
-C_{y p \alpha}(\mathcal{M}) \beta
\end{array}\right]\right. \\
& \left.+\left[\begin{array}{l}
-C_{N \delta}(\mathcal{M}) \delta_{r} \\
-C_{N \delta}(\mathcal{M}) \delta_{q}
\end{array}\right]\right)+m g\left[\begin{array}{c}
0 \\
\cos \theta
\end{array}\right]
\end{aligned}
$$

The external moments $M, N$ consist of pitch/yaw, Magnus, damping, and control terms:

$$
\begin{aligned}
{\left[\begin{array}{c}
M \\
N
\end{array}\right] } & =\bar{q} S d\left(\left[\begin{array}{l}
+C_{m \alpha}(\mathcal{M}, \alpha, \beta) \alpha \\
-C_{m \alpha}(\mathcal{M}, \alpha, \beta) \beta
\end{array}\right]+\frac{p_{a} d}{2 V}\left[\begin{array}{l}
C_{n p \alpha}(\mathcal{M}) \beta \\
C_{n p \alpha}(\mathcal{M}) \alpha
\end{array}\right]\right. \\
& \left.+\frac{d}{2 V}\left[\begin{array}{l}
C_{m q}(\mathcal{M}) q \\
C_{m q}(\mathcal{M}) r
\end{array}\right]+\left[\begin{array}{c}
C_{m \delta}(\mathcal{M}) \delta_{q} \\
-C_{m \delta}(\mathcal{M}) \delta_{r}
\end{array}\right]\right)
\end{aligned}
$$

The expressions of the forces and moments involve aerodynamic coefficients that depend in a nonlinear manner on the Mach number $\mathcal{M}=V / a(h)$, with $a$ the altitudedependent speed of sound. These coefficients are subject to uncertainties, as detailed in Table I. Other parameters and constants appearing in the expressions of the forces and moments are the dynamic pressure $\bar{q}=\frac{1}{2} \rho(h) V^{2}$, with $\rho(h)$ the altitude-dependent air density, the gravitational acceleration $g(h)$, the reference area $S$ and the calibre $d$.

The force and moment control terms involve virtual control variables $\left[\delta_{q}, \delta_{r}\right]^{T}$. These are related to the actual canard deflection angles $\left[\delta_{1}, \delta_{2}, \delta_{3}, \delta_{4}\right]^{T}$ through:

$$
\left[\begin{array}{l}
\delta_{q} \\
\delta_{r}
\end{array}\right]=T\left(\phi_{f}\right)\left[\begin{array}{cccc}
0 & \frac{1}{2} & 0 & -\frac{1}{2} \\
\frac{1}{2} & 0 & -\frac{1}{2} & 0
\end{array}\right]\left[\begin{array}{l}
\delta_{1} \\
\delta_{2} \\
\delta_{3} \\
\delta_{4}
\end{array}\right]
$$

with $T\left(\phi_{f}\right)=\left[\begin{array}{cc}\cos \phi_{f} & -\sin \phi_{f} \\ \sin \phi_{f} & \cos \phi_{f}\end{array}\right]$.

The relevant dynamics for flight control are the lateral (pitch/yaw) channels, which can be rewritten in the generic form:

$$
\begin{aligned}
\dot{\boldsymbol{x}}(t) & =f(\boldsymbol{x}(t), \boldsymbol{u}(t), \boldsymbol{\sigma}(t)) \\
\boldsymbol{y}(t) & =g(\boldsymbol{x}(t), \boldsymbol{u}(t), \boldsymbol{\sigma}(t))
\end{aligned}
$$

with states $\boldsymbol{x}=[\alpha, q, \beta, r]^{T}$, inputs $\boldsymbol{u}=\left[\delta_{q}, \delta_{r}\right]^{T}$, outputs $\boldsymbol{y}=\left[n_{z}, n_{y}, q, r\right]^{T}$, and parameters $\boldsymbol{\sigma}=\left[V, h, p_{a}, \theta\right]^{T}$. The load factors are defined as $\left[n_{z}, n_{y}\right]=\frac{1}{m g}\left[Z_{s}, Y_{s}\right]$, where $Z_{s}$ and $Y_{s}$ are respectively the normal and lateral components of the external forces excluding gravity (cf. Eq. (3)).
TABLE I

AERODYNAMIC COEFFICIENT UNCERTAINTIES

\begin{tabular}{|c|c|c|c|}
\hline Coefficient & Uncertainty (\%) & Coefficient & Uncertainty (\%) \\
\hline$C_{N \alpha}$ & 6 & $C_{m \alpha}$ & 3 \\
$C_{Y p \alpha}$ & 30 & $C_{n p \alpha}$ & 15 \\
$C_{N \delta}$ & 10 & $C_{m q}$ & 15 \\
- & - & $C_{m \delta}$ & 10 \\
\hline
\end{tabular}

The linearisation of the above nonlinear system requires defining an extended trimming vector $\boldsymbol{\rho}=\left[V, h, p_{a}, \theta, \alpha, \beta\right]^{T}$ so that equilibrium points can be analytically computed [4]. Because the trimming vector includes state variables, this leads to a quasi-LPV model:

$$
\begin{aligned}
& \dot{\boldsymbol{x}}_{\epsilon}(t)=\boldsymbol{A}(\boldsymbol{\rho}) \boldsymbol{x}_{\epsilon}(t)+\boldsymbol{B}(\boldsymbol{\rho}) \boldsymbol{u}_{\epsilon}(t) \\
& \boldsymbol{y}_{\epsilon}(t)=\boldsymbol{C}(\boldsymbol{\rho}) \boldsymbol{x}_{\epsilon}(t)+\boldsymbol{D}(\boldsymbol{\rho}) \boldsymbol{u}_{\epsilon}(t)
\end{aligned}
$$

where $\boldsymbol{x}_{\epsilon}, \boldsymbol{u}_{\epsilon}$, and $\boldsymbol{y}_{\epsilon}$ denote the state, input, and output deviation vectors from equilibrium. In anticipation of robustness analysis, aerodynamic uncertainties are introduced in the linearised model by computing corresponding linear fractional representations (LFR). This point is given more attention in Section IV.

In addition to the airframe model, the plant includes actuator and sensor dynamics. The canard actuators are modelled as second-order systems of natural frequency $20 \mathrm{~Hz}$ and damping ratio 0.781 , with an additional amplitude saturation $\delta_{\text {sat }}= \pm 10^{\circ}$. For simplicity, we use two virtual actuators corresponding to the two virtual command signals. The sensors are modelled as fast first-order systems with natural frequency $133 \mathrm{~Hz}$, and provide measurements of the load factors $\left[n_{z, m}, n_{y, m}\right]^{T}$, the angular rates $\left[q_{m}, r_{m}\right]^{T}$, and the deflection angles $\left[\delta_{q, m}, \delta_{r, m}\right]^{T}$. This amounts to a plant model of order $14=4+(2 \times 2)+(6 \times 1)$.

\section{Autopilot DesigN}

\section{A. Baseline Autopilot}

The baseline autopilot considered in this paper is identical to the one used in [2], and only its main features are recalled here. The controller is structured as in Fig.3, where the subscripts $\bullet_{c}$ and $\bullet_{m}$ denote commanded and measured variables respectively. In addition, symmetries are imposed on the controller gains, reflecting the symmetries of the plant:

$$
\begin{aligned}
\mathbf{K}_{i, e}=\left[\begin{array}{cc}
K_{i, e}^{(11)} & K_{i, e}^{(12)} \\
K_{i, e}^{(12)} & -K_{i, e}^{(11)}
\end{array}\right] & \mathbf{K}_{p, n}=\left[\begin{array}{cc}
K_{p, n}^{(11)} & K_{p, n}^{(12)} \\
K_{p, n}^{(12)} & -K_{p, n}^{(11)}
\end{array}\right] \\
\mathbf{K}_{p, \omega}=\left[\begin{array}{cc}
K_{p, \omega}^{(11)} & K_{p, \omega}^{(12)} \\
-K_{p, \omega}^{(12)} & K_{p, \omega}^{(11)}
\end{array}\right] & \mathbf{K}_{p, \delta}=\left[\begin{array}{cc}
K_{p, \delta}^{(11)} & K_{p, \delta}^{(12)} \\
K_{p, \delta}^{(12)} & -K_{p, \delta}^{(11)}
\end{array}\right]
\end{aligned}
$$

The eight gains are computed at $8 \times 6 \times 5=240$ operating points, using $\mathcal{H}_{\infty}$ multi-objective synthesis with systune [5]. The chosen design points correspond to an equidistant grid of a reduced three-dimensional flight envelope consisting of the scheduling variables $V \in[140 \mathrm{~m} / \mathrm{s}, 420 \mathrm{~m} / \mathrm{s}]$, $h \in[0 \mathrm{~m}, 15000 \mathrm{~m}]$, and $p_{a} \in[750 \mathrm{rad} / \mathrm{s}, 1600 \mathrm{rad} / \mathrm{s}]$. The global controller is obtained by linearly interpolating the gains between the design points. The gain surfaces remain relatively smooth, as shown in Fig.4. 


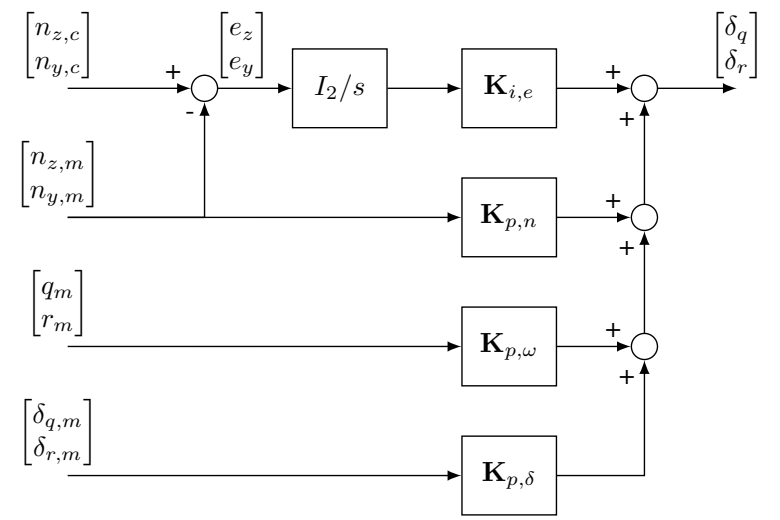

Fig. 3. Structure of the pitch/yaw channel linear controller

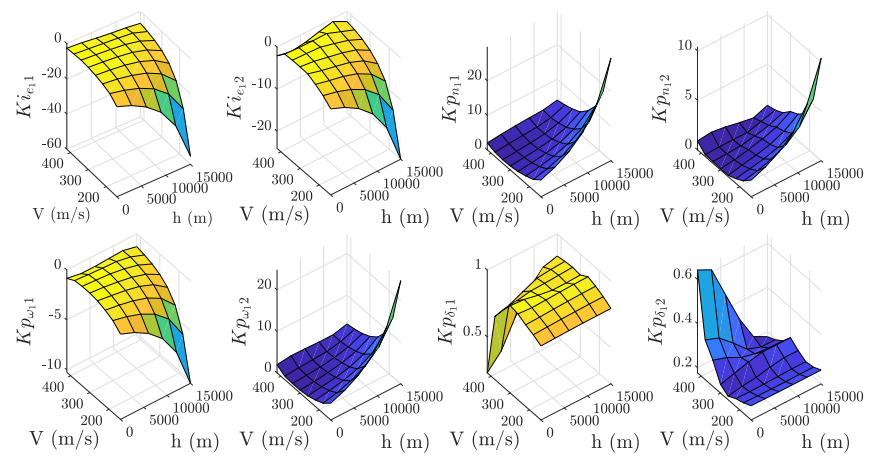

Fig. 4. Gain surfaces for fixed $p_{a}$

\section{B. Anti-Windup Synthesis}

To counteract the effects of saturations, an anti-windup compensator AW is added. As illustrated by Fig.5, this additional element is driven by the difference between the desired controller output $y_{c}$ and the achieved command signal $u=\operatorname{sat}\left(y_{c}\right)$. It then generates a signal $v=\left[v_{1}, v_{2}\right]^{T}$ that modifies the controller dynamics. In an LTI context, this yields:

$$
(K)\left\{\begin{array}{l}
\dot{x}_{c}=A_{c} x_{c}+B_{c}\left[\begin{array}{c}
y \\
w
\end{array}\right]+v_{1} \\
y_{c}=C_{c} x_{c}+D_{c}\left[\begin{array}{c}
y \\
w
\end{array}\right]+v_{2}
\end{array}\right.
$$

where $\left(A_{c}, B_{c}, C_{c}, D_{c}\right)$ is a state-space realisation of the baseline controller $K$.

In the context of the studied application, we assume a fixed roll angle $\phi_{f}=45^{\circ}$ and $\delta_{p}=0$ during the guided phase (which can be ensured by the independent roll autopilot from [6]). In addition, a pseudo-inverse allocation is used to convert the virtual controller outputs $\left[\delta_{q}, \delta_{r}\right]^{T}$ back into the commanded canard angles $\left[\delta_{1}, \delta_{2}, \delta_{3}, \delta_{4}\right]^{T}$. This yields:

$$
\left[\begin{array}{l}
\delta_{1} \\
\delta_{2} \\
\delta_{3} \\
\delta_{4}
\end{array}\right]=\frac{\sqrt{2}}{2}\left[\begin{array}{cc}
0 & -1 \\
+1 & 0 \\
0 & +1 \\
-1 & 0
\end{array}\right]\left[\begin{array}{l}
\delta_{q}+\delta_{r} \\
\delta_{q}-\delta_{r}
\end{array}\right]
$$

From the above relation, it follows that the amplitude saturation $\delta_{\text {sat }}=10^{\circ}$ applied on the canard angles can be expressed as a saturation $\delta_{\text {sat,virtual }}=\sqrt{2} \times 10^{\circ}$ on $\left[\delta_{q}+\delta_{r}, \delta_{q}-\delta_{r}\right]^{T}$, which is subsequently defined as the controller output $y_{c}$ with respect to Eq. (9) and Fig.5.

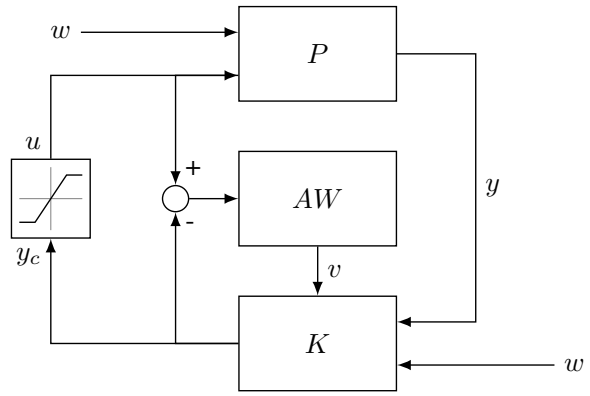

Fig. 5. Principle of anti-windup compensation

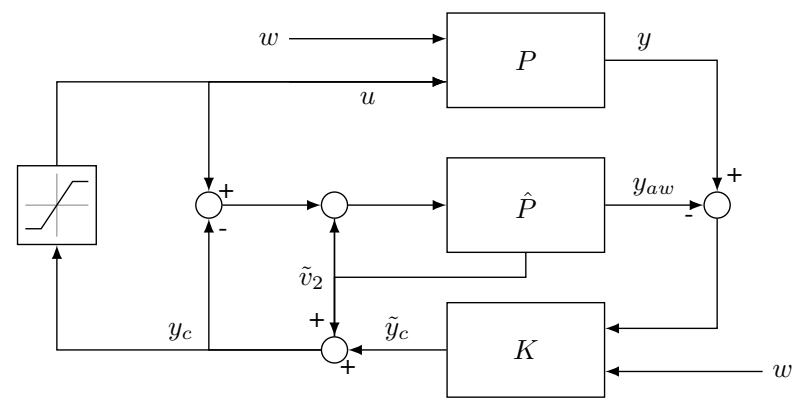

Fig. 6. MRAW compensation scheme

In [2], local static anti-windup compensators were computed and then interpolated to cover the flight envelope. While the simplicity of this structure makes it attractive, it is interesting to investigate whether we can benefit from the increased number of degrees of freedom of a dynamic anti-windup. In this study, a model-recovery anti-windup (MRAW) approach is proposed (see [7], [8]). The principle of MRAW augmentation is shown in Fig.6, and consists in embedding a model of the plant $P$ into the anti-windup. Noting $\left(A_{p}, B_{p}, C_{p}, D_{p}\right)$ a realisation of the plant with removed exogenous input and output, we have

$$
(\hat{P})\left\{\begin{array}{c}
\dot{x}_{a w}=A_{p} x_{a w}+B_{p}\left(u-y_{c}+\tilde{v}_{2}\right) \\
y_{a w}=C_{p} x_{a w}+D_{p}\left(u-y_{c}+\tilde{v}_{2}\right) \\
\tilde{v}_{2} \text { to be designed }
\end{array}\right.
$$

The motivation for this structure is that $x_{a w}$ tracks the mismatch between the unconstrained response and the closedloop response. A natural objective is then to bring $x_{a w}$ to 0 , in which case the unconstrained behaviour is recovered. This is achieved by way of the signal $\tilde{v}_{2}$. Note that the antiwindup signals $y_{a w}$ and $\tilde{v}_{2}$ in the MRAW scheme do not exactly match the signals $v_{1}$ and $v_{2}$ from Eq. (9). However, these can directly be derived from:

$$
v_{1}=-B_{c} y_{a w} \quad v_{2}=-D_{c} y_{a w}+\tilde{v}_{2}
$$

Several algorithms exist to compute MRAW compensators, and we refer to [7] and [8] for more details on the topic. Because the flight envelope includes points where the plant is exponentially unstable, and to keep the tuning effort to a minimum, a regional LQ-based MRAW was chosen. Thus the anti-windup signal $\tilde{v}_{2}$ is given by:

$$
\tilde{v}_{2}=K_{l q r} x_{a w}
$$



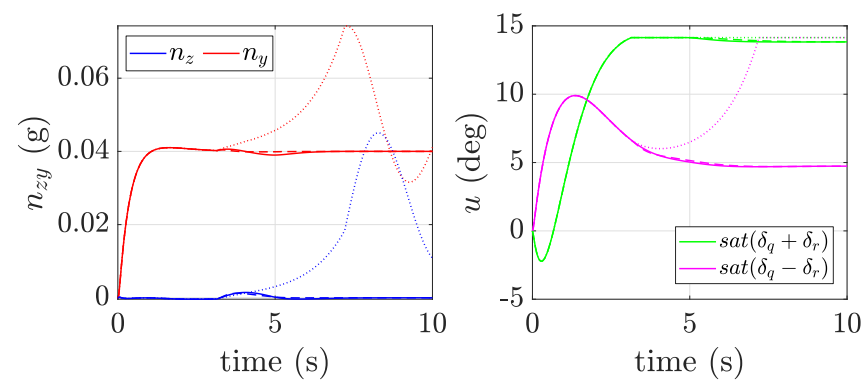

Fig. 7. Response to a step input on $n_{y}$ : without anti-windup (dotted), with static anti-windup (dashed), and with MRAW-LQ (full)

associated to the quadratic cost function $J=$ $\int_{0}^{\infty}\left(x_{a w}^{T} Q x_{a w}+\tilde{v}_{2} R \tilde{v}_{2}\right) d t$. The corresponding gain matrix $K_{\text {lqr }} \in \mathbb{R}^{2 \times 14}$ is thus easily computed (e.g. with the Matlab command $\operatorname{Klqr}=-\operatorname{lqr}(A, B, Q, R)$; the minus sign arises from the sign convention of the antiwindup signals). The cost function is kept the same for all the 240 design points, with $Q=\operatorname{diag}\left(I_{10}, 50 \cdot I_{4}\right)$ and $R=I_{2}$, enforcing a stronger penalty on the four last states corresponding to the (mismatch on the) actuator states, see Section II. The resulting compensator is capable of improving step responses, as illustrated by Fig.7. On this particular case, there is no apparent advantage in using the LQ-based MRAW compensator compared to the static one. The benefits will become clearer when robustness analysis will be addressed in Section IV (see Fig. 10). It is worth noting that the 240 local MRAW compensators were calculated in about 2 seconds. By contrast, obtaining the static anti-windup compensators took 152 seconds. The relative length of the latter computation can be attributed to the required LMI resolution, although it should be pointed out that it was done using LMI Lab, and that faster solvers are available.

The relatively high order $n_{a w}=14$ of the compensators does not lend itself to the interpolation of the state-space coefficients. Instead, the interpolation method chosen to obtain the global compensator is based on output blending as described in [9]: at a given operating point, the neighbouring local anti-windup compensators are made active, and their outputs are interpolated to yield the global compensator output. Since three scheduled variables $\left(V, h, p_{a}\right)$ are considered, the interpolation is made between the outputs of $2^{3}=8$ local anti-windup compensators. Although this interpolation scheme does not offer stability or performance guarantees, it remains attractive as its implementation is straightforward even for high order systems. Furthermore, it involves fewer on-line operations compared to other ad hoc strategies such as pole-zero or state-space interpolations.

\section{RoBustness AnALYSIS USING IQCS}

\section{A. IQC Framework}

The anti-windup synthesis conducted above was done assuming a nominal plant model and did not directly address robustness issues. The robustness analysis of the closedloop can be done using integral quadratic constraints (IQCs). The main principles of the method are briefly summed up here, and we refer to [10] and [11] for more thorough
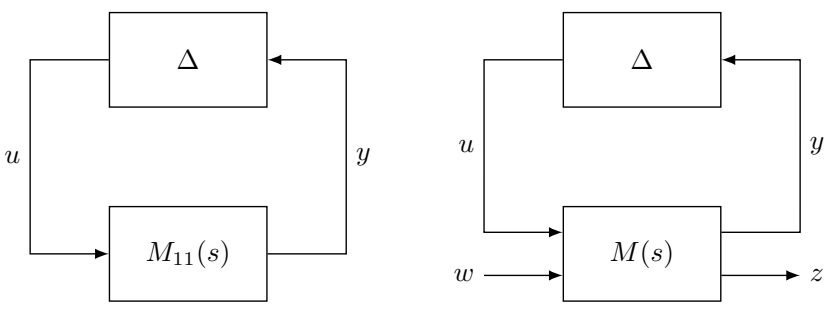

Fig. 8. LFR for IQC analysis (left: stability; right: performance)

theoretical explanations. IQC-based robustness analysis relies on modelling the system to analyse as an LFR, as depicted in Fig.8. The system $M(s)=\left[\begin{array}{ll}M_{11}(s) & M_{12}(s) \\ M_{21}(s) & M_{22}(s)\end{array}\right]$ is the nominal model, assumed to be stable, and $\Delta \in \boldsymbol{\Delta}$ is a causal bounded operator that gathers the uncertainties and nonlinearities of the system. A so-called IQC multiplier $\Pi: j \mathbb{R} \rightarrow \mathbb{C}^{\left(n_{y}+n_{u}\right) \times\left(n_{y}+n_{u}\right)}$ is then sought, whose role is roughly to capture the properties of $\Delta$ and to provide a stability certificate. These notions are made precise by the main stability theorem from [10]:

Theorem 1: Let $M_{11}(s)$ be stable and $\Delta \in \Delta$. If the following properties hold:

- well-posedness: for every $\tau \in[0,1], I-\tau M_{11}(s) \Delta$ has a causal inverse;

- for every $\tau \in[0,1], \tau \Delta$ satisfies the IQC defined by the multiplier $\Pi$, i.e.:

$$
\forall y \in \mathcal{L}_{2} \quad \int_{-\infty}^{+\infty}\left[\begin{array}{l}
\hat{y}(j \omega) \\
\hat{u}(j \omega)
\end{array}\right]^{*} \Pi(j \omega)\left[\begin{array}{l}
\hat{y}(j \omega) \\
\hat{u}(j \omega)
\end{array}\right] d \omega \geq 0
$$

with $u=\tau \Delta(y)$, and where $\mathcal{L}_{2}$ is the space of signals with finite energy, and for $f \in \mathcal{L}_{2}, \hat{f}$ denotes the Fourier transform of $f$.

- there exists $\epsilon>0$ such that

$$
\forall \omega \in \mathbb{R}\left[\begin{array}{c}
M_{11}(j \omega) \\
I
\end{array}\right]^{*} \Pi(j \omega)\left[\begin{array}{c}
M_{11}(j \omega) \\
I
\end{array}\right] \preceq-\epsilon I
$$

then the interconnection of $M_{11}(s)$ and $\Delta$ is stable.

The numerous works on IQC theory provide multipliers for a wide variety of uncertainties. As emphasised in [11] and [12], it is particularly convenient to work with multipliers parametrised as $\Pi=\Psi^{*} P \Psi$ with some fixed $\Psi \in R H_{\infty}$ and $P=P^{T} \in \boldsymbol{P}$, where $\boldsymbol{P}$ is described by LMI constraints. Indeed, application of the Kalman-Yakubovich-Popov lemma then allows to replace the condition of Eq. (15) by the equivalent condition:

- there exists $X=X^{T}$ and $P \in \boldsymbol{P}$ such that

$$
\left[\begin{array}{cc}
I & 0 \\
A & B \\
C & D
\end{array}\right]^{T}\left[\begin{array}{ccc}
0 & X & 0 \\
X & 0 & 0 \\
0 & 0 & P
\end{array}\right]\left[\begin{array}{cc}
I & 0 \\
A & B \\
C & D
\end{array}\right] \prec 0
$$

where $(A, B, C, D)$ is a minimal realisation of $\Psi(s)\left[\begin{array}{c}M_{11}(s) \\ I\end{array}\right]$.

Thus verifying stability of the interconnection boils down to solving an LMI problem.

The IQC framework extends seamlessly to performance analysis, by taking into account the performance channel 


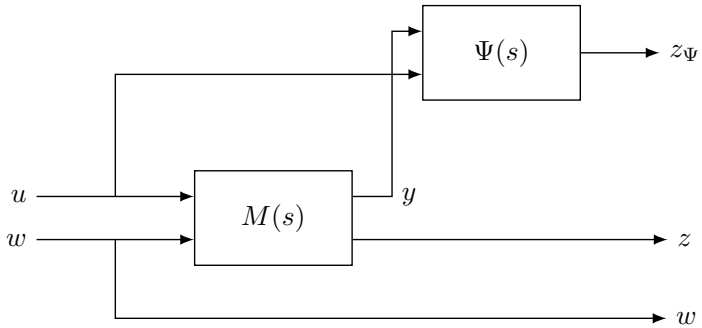

Fig. 9. Augmented system for IQC analysis

$w \rightarrow z$ from Fig. 8. In particular, when evaluating performance through the $\mathcal{L}_{2}$-gain, Eq. (16) is adapted into:

$$
\left[\begin{array}{ll}
I & 0 \\
\mathcal{A} & \mathcal{B} \\
\mathcal{C} & \mathcal{D}
\end{array}\right]^{T}\left[\begin{array}{ccc}
0 & X & 0 \\
X & 0 & 0 \\
0 & 0 & \mathcal{P}
\end{array}\right]\left[\begin{array}{ll}
I & 0 \\
\mathcal{A} & \mathcal{B} \\
\mathcal{C} & \mathcal{D}
\end{array}\right] \prec 0
$$

where $(\mathcal{A}, \mathcal{B}, \mathcal{C}, \mathcal{D})$ is a minimal realisation of the system in Fig.9 and $\mathcal{P}=\operatorname{diag}\left(P, I_{z},-\gamma^{2} I_{w}\right)$. We then seek matrices $X=X^{T}$ and $\mathcal{P}$ that minimise $\gamma$, under the LMI constraints (17) and $P \in \boldsymbol{P}$. This provides an upper bound on the worstcase $\mathcal{L}_{2}$ gain from $w$ to $z$.

\section{B. Application to the Guided Projectile}

To perform IQC analysis, an LFR model of the projectile linearised pitch/yaw channels is computed at an operating point of the flight envelope corresponding to high velocity and low altitude. This step is done using the GSS library of the SMAC toolbox [13]. The resulting $\Delta$ block has the following structure:

$$
\begin{gathered}
\Delta=\operatorname{diag}\left(\delta_{C_{N \alpha}} I_{2}, \delta_{C_{Y p \alpha}} I_{2}, \delta_{C_{N \delta}} I_{2}, \delta_{C_{m \alpha}} I_{2}, \delta_{C_{n p \alpha}} I_{2},\right. \\
\left.\delta_{C_{m q}} I_{2}, \delta_{C_{m \delta}} I_{2}, \mathrm{dz}, \mathrm{dz}\right)
\end{gathered}
$$

with normalised aerodynamic uncertainties (i.e. each $\delta_{C_{i}}$ lies in $[-1,1])$, and where the deadzone nonlinearities $\mathrm{dz}$ arise from a rewriting of the saturation functions: $\mathrm{dz}(x)=$ $x-\operatorname{sat}(x)$. The performance channel $w \rightarrow z$ is taken as the transfer from the guidance signals $\left[n_{z, c}, n_{y, c}\right]^{T}$ to the measured load factors $\left[n_{z, m}, n_{y, m}\right]^{T}$.

A first analysis can be done ignoring the parametric uncertainties. The remaining two deadzone nonlinearities can then be locally described as sector bounded slope-restricted nonlinearities $\phi \in \sec (0, \beta) \cap \operatorname{slope}(0, \beta)$. Accordingly, we use a combination of full-block circle criterion and ZamesFalb multipliers (see Class 13 and Class 15 in [11] for their implementation) to formulate the LMI problem to solve. Fig. 10 shows bounds $\gamma$ on the $\mathcal{L}_{2}$-gain plotted over the sector slope $\beta$, for different control strategies. It can be observed that anti-windup compensators increase the maximum value of the parameter $\beta$ for which stability can be guaranteed, which is especially pronounced for the LQ-based MRAW. Although none of the design is able to validate stability for $\beta=1$, and thus for the actual deadzone nonlinearities, this still qualitatively hints at better stability properties of the MRAW scheme at this operating point. Curiously, there seems to be a slight performance degradation for small values of $\beta$ when using the static anti-windup, compared to the configuration with no anti-windup.

Next, a robustness analysis including the aerodynamic uncertainties, i.e. corresponding to the $\Delta$ block structure (18)

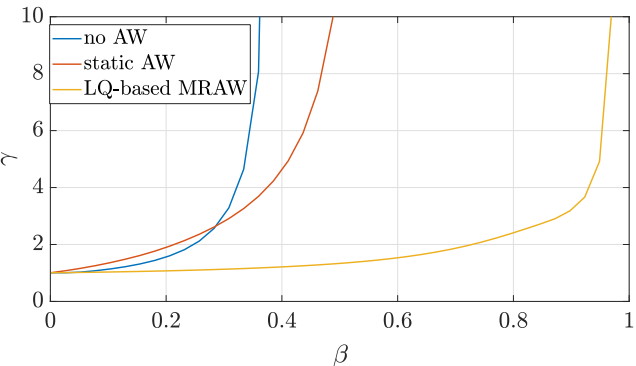

Fig. 10. Bounds on the $\mathcal{L}_{2}$-gain in the presence of a nonlinearity $\phi \in$ $\sec (0, \beta)^{2} \cap \operatorname{slope}(0, \beta)^{2}$

is performed. This is done by adding dynamic $D G$-scaling multipliers (see Class 5 in [11]). Results with the LQ-based MRAW are shown in Fig. 11, where for convenience the amplitudes of all the aerodynamic uncertainties are made to vary simultaneously. Analyses were conducted on a $20 \times 20$ grid, each one involving 2250 decision variables. The average resolution time was $12 \mathrm{~s}$ using MOSEK/YALMIP [14], [15]. The difficulty of validating performance against parametric uncertainties for higher values of $\beta$ is clearly seen by the increasing steepness of the surface in this area. This also illustrates how IQC analysis can be used to analyse the sensitivity of the system to specific groups of uncertainties.

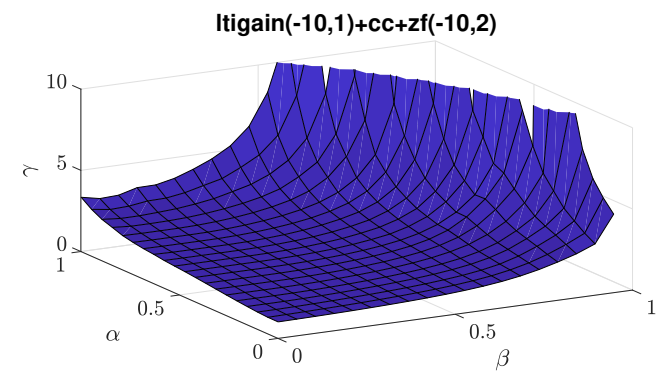

Fig. 11. Bounds on the $\mathcal{L}_{2}$-gain in the presence of aerodynamic uncertainties $\left|\delta_{C_{i}}\right| \leq \alpha$ and nonlinearity $\phi \in \sec (0, \beta)^{2} \cap \operatorname{slope}(0, \beta)^{2}$

\section{Simulation Results}

To evaluate the autopilot design, 300 Monte Carlo trajectory simulations are conducted, with uncertainties applied to each aerodynamic coefficient. The sampling of these coefficients is done assuming a normal distribution, where the standard deviation $\sigma$ is such that $3 \sigma$ corresponds to maximum uncertainty as reported on Table I. For simplicity, the sampled uncertainty level is kept constant over the entire flight trajectory.

Simulations are first run on the closed-loop nonlinear model with no saturations. As seen on Fig. 12 (for better visibility, only 30 simulation results are shown), the baseline autopilot is able to cope with aerodynamic uncertainties and to follow the guidance signals. This results in a centimetric accuracy at impact in all the tested cases. However, 49 of these trajectories (about 16\% of the cases) require canard deflection angles above $10^{\circ}$. With active saturations, this can lead to a dramatic degradation of the guided phase, as shown with the trajectory featured on Fig. 13, associated to a miss distance of $74 \mathrm{~m}$. Fig. 14 shows how anti-windup augmentation can recover a satisfactory behaviour. Fig.15 

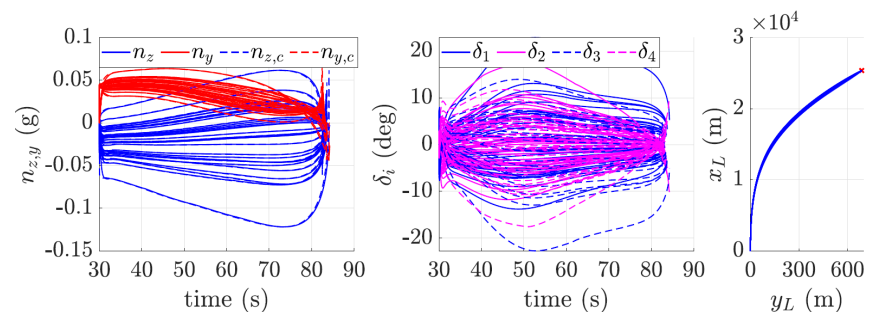

Fig. 12. Monte Carlo trajectories without saturations; normal and lateral accelerations (left), canard deflection angles (middle), trajectory (right)
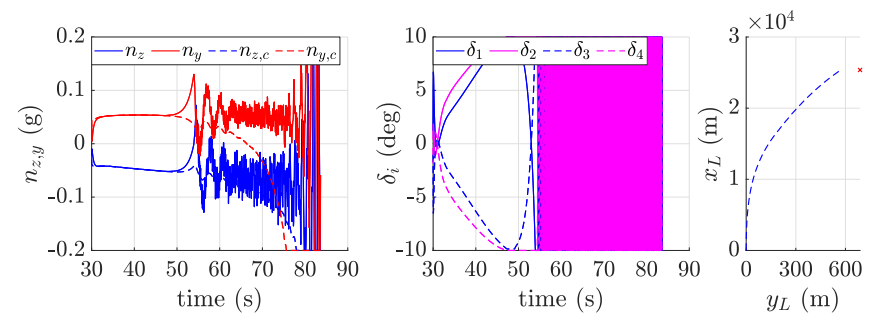

Fig. 13. Trajectory with saturations; normal and lateral accelerations (left), canard deflection angles (middle), trajectory (right)

shows the miss distance corresponding to the 49 identified cases with saturations, for different projectile configurations: ballistic, with only the baseline autopilot, and with antiwindup compensation. The iterations are sorted by increasing order of the miss distance for the baseline configuration. The benefit of anti-windup is apparent from this figure, with an increased number of cases where the target is successfully reached (28 out of 49). Although very large miss distance can still occur even with anti-windup, the dispersion is noticeably reduced. Focusing on these 49 cases, the average miss distance in the baseline configuration is $100.7 \mathrm{~m}$, against $21.8 \mathrm{~m}$ with anti-windup. It is worth emphasising that this improvement is obtained with an LQ-based MRAW that has not specifically been tuned for robust performance.

\section{CONCLUSION}

This paper deals with the autopilot design of a guided dual-spin projectile. In particular, the use of a relatively high order anti-windup compensator is investigated. IQC analysis is set up in a fixed-parameter framework, giving the possibility to assess robustness properties, compare different autopilot designs, and analyse the sensitivity of the system to the various uncertainties. The integration of the antiwindup compensator in the nonlinear, parameter-varying model is done with controller output blending. This approach is validated through Monte Carlo simulations, and opens up the possibility to investigate other dynamic schemes. The results show that the resulting control law is able to mitigate the performance degradation induced by aerodynamic uncertainties.

Future work will focus on exploiting IQC analysis results to help with further autopilot syntheses, encompassing tuning and controller order reduction, with the aim to further improve robust performance. To this end, refinements of the IQC analysis are still possible. The LFR model could include time-varying elements to increase its representativeness with respect to the complete nonlinear model. In addition, more representative profiles of the signals could be better taken
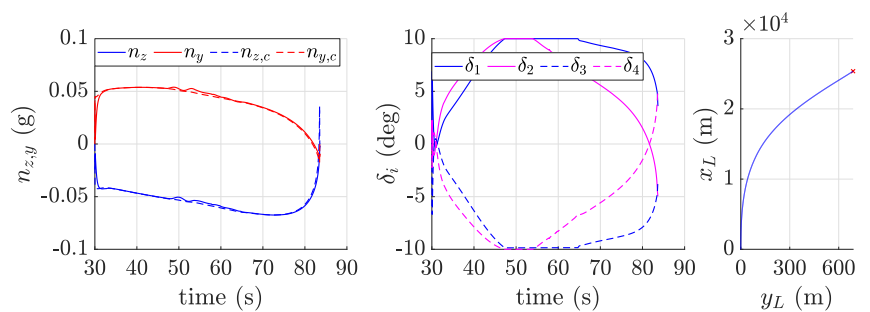

Fig. 14. Trajectory with saturations and anti-windup; normal and lateral accelerations (left), canard deflection angles (middle), trajectory (right)

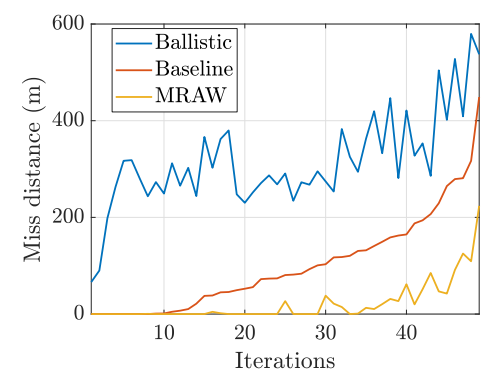

Fig. 15. Miss distance distribution for 49 trajectories where uncertainty samples on aerodynamic coefficients lead to saturations

into account to define a more relevant performance index than the rather conservative $\mathcal{L}_{2}$ gain.

\section{REFERENCES}

[1] F. Sève, S. Theodoulis, P. Wernert, M. Zasadzinski, and M. Boutayeb, "Gain-scheduled $\mathcal{H}_{\infty}$ loop-shaping autopilot design for spin-stabilized canard-guided projectiles," AerospaceLab Journal, 2017.

[2] S. Thai, S. Theodoulis, C. Roos, J.-M. Biannic, and M. Proff, "Gain-scheduled autopilot design with anti-windup compensator for a dual-spin canard-guided projectile," in Proceedings of the 4th IEEE Conference on Control Technology and Applications, 2020.

[3] R. McCoy, Modern Exterior Ballistics: The Launch and Flight Dynamics of Symmetric Projectiles. Schiffer, 1999.

[4] F. Sève, S. Theodoulis, P. Wernert, M. Zasadzinski, and M. Boutayeb, "Flight dynamics modeling of dual-spin guided projectiles," IEEE Transactions on Aerospace and Electronic Systems, 2017.

[5] P. Apkarian and D. Noll, "Nonsmooth $H_{\infty}$ synthesis," IEEE Transactions on Automatic Control, 2006.

[6] S. Thai, S. Theodoulis, C. Roos, and J.-M. Biannic, "Robust design for the roll-channel autopilot of a canard-guided dual-spin projectile," IFAC-PapersOnLine, 2019, 21st IFAC Symposium on Automatic Control in Aerospace ACA 2019.

[7] S. Galeani, S. Tarbouriech, M. Turner, and L. Zaccarian, "A tutorial on modern anti-windup design," European Journal of Control, 2009.

[8] L. Zaccarian and A. R. Teel, Modern Anti-windup Synthesis: Control Augmentation for Actuator Saturation. Princeton Series in Applied Mathematics, 2011.

[9] J. Kelly and J. H. Evers, An interpolation strategy for scheduling dynamic compensators, 1997.

[10] A. Megretski and A. Rantzer, "System analysis via integral quadratic constraints,' IEEE Transactions on Automatic Control, vol. 42, no. 6, pp. 819-830, 1997.

[11] J. Veenman, C. W. Scherer, and H. Köroğlu, "Robust stability and performance analysis based on integral quadratic constraints," European Journal of Control, vol. 31, pp. 1 - 32, 2016.

[12] M. Fetzer, C. Scherer, and J. Veenman, "Invariance with dynamic multipliers," IEEE Transactions on Automatic Control, vol. 63, pp. 1929-1942, 2018.

[13] J.-M. Biannic and C. Roos, "Generalized state space: a new Matlab class to model uncertain and nonlinear systems as linear fractional representations," 2016-2019. [Online]. Available: http://w3.onera.fr/smac/gss

[14] M. ApS, The MOSEK optimization toolbox for MATLAB manual. Version 9.0, 2019. [Online]. Available: http://docs.mosek.com/9.0/ toolbox/index.html

[15] J. Lofberg, "YALMIP: A toolbox for modeling and optimization in Matlab," in Proceedings of the 2004 IEEE International Conference on Robotics and Automation, 2004, pp. 284-289. 